\title{
Low dimensional reaction kinetics and self-organization
}

\author{
R. Kopelman *, L.W. Anacker, E. Clement, L. Li and L. Sander \\ Departments of Chemistry and Physics, The University of Michigan, Ann Arbor, MI 48109 (U.S.A.)
}

(Received 8 November 1989; accepted 23 February 1990)

\begin{abstract}
Kopelman, R., Anacker, L.W., Clement, E., Li, L. and Sander, L., 1991. Low dimensional reaction kinetics and self-organization. Chemometrics and Intelligent Laboratory Systems, 10: 127-132.

Diffusion-limited reaction kinetics becomes anomalous not only for fractals, with their anomalous diffusion, but also for low-dimensional (one and two) and disperse media, where the random walk is compact. We focus on annihilation, recombination and trapping reactions under non-equilibrium steady state (steady source) or batch (big bang) conditions. The typical reactions are: $\mathbf{A}+\mathbf{A} \rightarrow$ Products, $\mathbf{A}+\mathbf{B} \rightarrow$ Products and $\mathbf{A}+\mathbf{C} \rightarrow$ Products. We are interested in the global rate laws, and their relation to particle-particle distributions (e.g., pair-correlation and nearest-neighbor distribution functions) and in local rate laws (if definable). Anomalous reaction kinetics (more than classical kinetics) is particularly sensitive to initial conditions, source term structure, conservation laws (e.g., equal densities for $\mathbf{A}$ and B), excluded volume effects, and medium size, dimensionality and anisotropy. Analytical formalisms, scaling arguments, computer (and supercomputer) simulations and experiments (on chemical and physical reactions) all play an important role in the newly emerging picture.
\end{abstract}

\section{INTRODUCTION}

This work can be viewed as a natural extension of the activity dealing with relaxation phenomena and transient kinetics problems in disordered media [1-4]. Its domain of application spans various areas of the physics and chemistry of condensed matter. For example, reactions of the type $\mathrm{A}+\mathrm{A} \rightarrow \mathbf{0}$ or $\mathrm{A}+\mathrm{T} \rightarrow \mathrm{T}$ are models describing exciton kinetics in disordered molecular crystals or polymer blends. Reactions of the type $A+B \rightarrow$ 0 are found in solid state physics in the case of electron-hole annihilation or defect fusion. A combination of experiments and Monte-Carlo simulations [5] has paved the way for a new theo- retical understanding of steady-state rate laws and the kinetic self-organization of atoms, defects and elementary excitations in low dimensional media. This theory is presented below.

Diffusion limited trapping is of particular interest in studies of energy migration and luminescence $[1,5]$. We present below some new simulations and their relation to theory. This includes both rate laws and self-ordering. Of particular interest is the resulting anomalously high partial order of reactions as a function of trap concentration.

'Big-bang' reaction models are simpler than steady-source models. The pioneering work has been done by Ovchinnikov and Zeldovich [6] and 
by Toussaint and Wilczek [7], with applications to fractals by Klafter et al. [8], Kang and Redner [9] and Klymko and Kopelman [10]. However, these ignored both finite size effects and finite correlation effects (at time zero). We demonstrate here that these finite extent effects give rise to new scaling effects, i.e., anomalous time exponents and reaction orders. In particular, for the $A+B$ reaction in one-dimension the time exponent rises from $1 / 4$ (Zeldovich value) to $3 / 4$ or 1 (depending on boundary conditions).

\section{THEORY: STEADY-STATE DIFFUSION CONTROLLED} BIMOLECULAR REACTIONS

In the classical picture, all bimolecular reactions are the same and the distribution of reactants is at random. Also, the reaction rate is proportional to the product of the reactant densities (overall order of reaction $X=2$ ). Previous works show that the time dependence of such reactive systems, relaxing from an initial random situation, exhibits anomalous decay rates in low dimensions due to local fluctuations in reactant density [6-9]. Here we report the results of a theoretical investigation on the steady state properties of three different bimolecular diffusion limited reactions, taking place on regular Euclidean spaces and on fractal structures [11-13]. We show that the relevant parameter describing the steady state of the reaction kinetics is the spectral dimension $d_{\mathrm{s}}$. The spectral dimension is an intrinsic parameter characterizing energy transfer properties, and in particular, diffusion in a medium. For Euclidean structures, $d_{\mathrm{s}}$ is the Euclidean dimension $d$, and the case of Euclidean spaces is viewed as an extension of the fractal case when we take $d=d_{\mathrm{s}}$. The reason for the influence of the spectral dimension on reaction kinetics is due to the fact that $d_{\mathrm{s}}$ controls the time dependence of the number of distinct sites visited by a random walker. For spectral dimension $d_{\mathrm{s}}<2$ we show that a bimolecular reaction induces a selforganization of reactants up to a scale $\Lambda$ such that:

$\Lambda \approx \tau^{1-d_{s} / 2} \quad d_{s}<2$

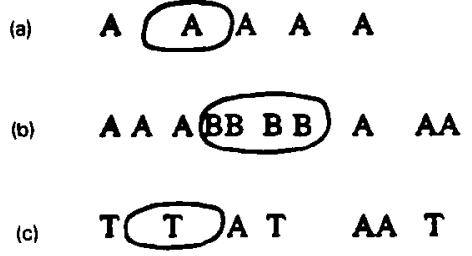

Fig. 1. Schematic representation of the three cases of self organization on a one-dimensional system. The circled domains represented here are of the order of $\Lambda$, the self organization scale. 1(a) is a depletion in the $A+A \rightarrow 0$ case. 1(b) is a segregation in the $A+B \rightarrow 0$ case. 1 (c) is a trap-particle depletion in the $A+T \rightarrow T$ case.

where $\tau$ is a characteristic time which is situation dependent. For $d_{\mathrm{s}}>2, \Lambda$ is microscopic and independent of $\tau$, therefore no large scale structure exists and the reaction kinetics is classical. The case $d_{\mathrm{s}}=2$ is found to be the critical dimension of the problem, where we find a marginal logarithmic dependence of $\Lambda$ with $\tau$. Below the critical dimension, large scale density fluctuations become relevant and each situation has its own phenomenology (see Fig. 1). In particular, we may find macroscopic reaction laws with anomalous reaction orders (larger than 2) or anomalous rate constants. In all the cases investigated we found that the scaling behavior of the self organization length can be case in an interesting general way. For every dimension we can write:

$\Lambda / a \approx S_{\tau} / V_{\tau}$

where $a$ is the microscopic scale, $S_{\tau}$ is the volume effectively explored by a particle during the time $t$ (number of distinct sites visited) and $V_{\tau}$ is the total (cumulative) volume swept out (proportional to $\tau$ ).

In bimolecular diffusion limited processes the overall balance between reaction rates and steady state densities is accounted for by the Smoluchowski boundary condition:

$Q \approx\left(\rho_{1} \rho_{2}\right) / \Lambda$

where $\rho_{1}$ and $\rho_{2}$ are, respectively, the steady state densities of reactants 1 and 2 ( 1 and 2 can be identical species). The scaling dependence of the self-organization scale $\Lambda$ on $\tau$ is at the origin of the non-classical behavior. 
In the case of homomolecular annihilation, A $+\mathrm{A} \rightarrow 0, \Lambda$ is a typical scale of depletion around each reactant and $\tau$ is the typical reactant life-time with:

$\tau=\rho / Q$

where $\rho$ is the steady state density of $A$. We obtain an anomalous effective reaction order:

$X=1+2 / d_{\mathrm{s}} \quad d_{\mathrm{s}}<2$

In the case of heteromolecular annihilation, $\mathrm{A}+\mathrm{B} \rightarrow 0, \lambda$ is the scale of a self-organization phenomenon called segregation. At steady state, domains of identical species with sizes comparable to $\lambda$ build up in the medium. The situation is more complex than in the homomolecular reaction case and $\tau$ is found to be dependent either on source conditions or on some intrinsic particle life time. We separated the source terms into two main categories. In the first category we consider sources for which at any time an identical number of As and Bs is conserved in the medium. If reactants are created at random, we find:

$\tau \approx L^{2}$

where $L$ is the system size. We observe a size dependent segregation. With the same conservation constraint, if the particles are created as A-B pairs with $A$ and $B$ separated by a distance $\delta$, we have:

$\tau \approx \delta^{2}$

The segregation scale becomes dependent on $\delta$. It is important to notice that for geminate creation, we obtain a microscopic segregation scale and this situation becomes analogous to classical kinetics. In the second category, we consider sources where the conservation constraint is removed. If no other decay mechanism is present, fluctuations in particle difference grow until we have a complete saturation of the loop with one of the species. There is no reactive steady state. If an extra (first order) decay mechanism is considered, fluctuations grow up to a size defined by the intrinsic lifetime of the decay mechanism. In particular if we consider vertical annihilation with an external rate of particles $R$ we have:

$\tau=R^{-1}$
In this case we obtain at low density an effective reaction order:

$X=4 / d_{\text {s }}$

On the other hand, if the decay is controlled by an intrinsic mechanism $\mathrm{A} \rightarrow 0$ and $\mathrm{B} \rightarrow 0$, with the same rate constant $K$, then we have

$\tau \approx K^{-1}$

We induce a $K$ dependent segregation but no anomalous reaction order. These last three cases are important for practical applications because, besides geminate particle creation, it is difficult to find a source satisfying the exact conservation constraint. However, though the conservation is not exact, these cases lead to a mesoscopic segregation (or a total saturation).

For the trapping problem, $A+T \rightarrow T$, the fluctuation of the trap distribution is found to be unimportant for the leading scaling behavior of the self organization length $\Lambda$. The relevant fact is that we have, for $d_{\mathrm{s}}<2$, an organization of particles $\mathrm{A}$ around the traps. The typical lifetime at steady state is:

$\tau \approx \rho / Q$

with $\rho$ the density of $\mathrm{A}$ and $Q$ the reaction rate. The scale of the trap-particle organization is:

$\Lambda \approx c^{1-2 / d_{\mathrm{s}}}$

where $c$ is the trap concentration. We have the anomalous rate law:

$Q \approx \rho c^{2 / d_{\mathrm{s}}}$

with an anomalous order relatively to the trap concentration:

$X=2 / d_{\mathrm{s}}$

and we note that the overall reaction order is $1+2 / d_{2}$, the same as for the $\mathrm{A}+\mathrm{A} \rightarrow 0$ case.

\section{SIMULATIONS OF STEADY-STATE TRAPPING}

We tested the trapping eq. (3). The Monte-Carlo simulations at the John von Neumann National Supercomputer Center give, for the Sierpinski gasket, a partial order $Y=1.02 \pm 0.02$, with respect to the particle density $\rho$, and a partial order 
(a)

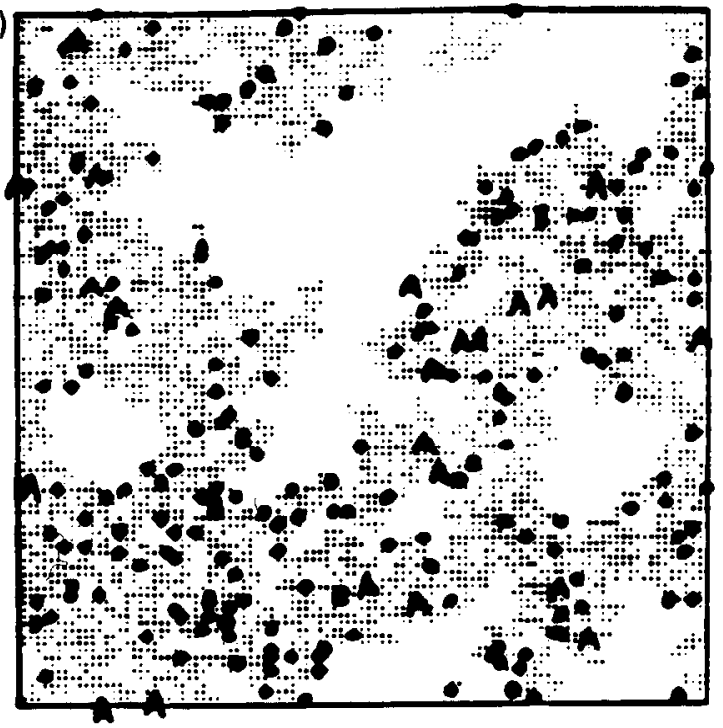

(b

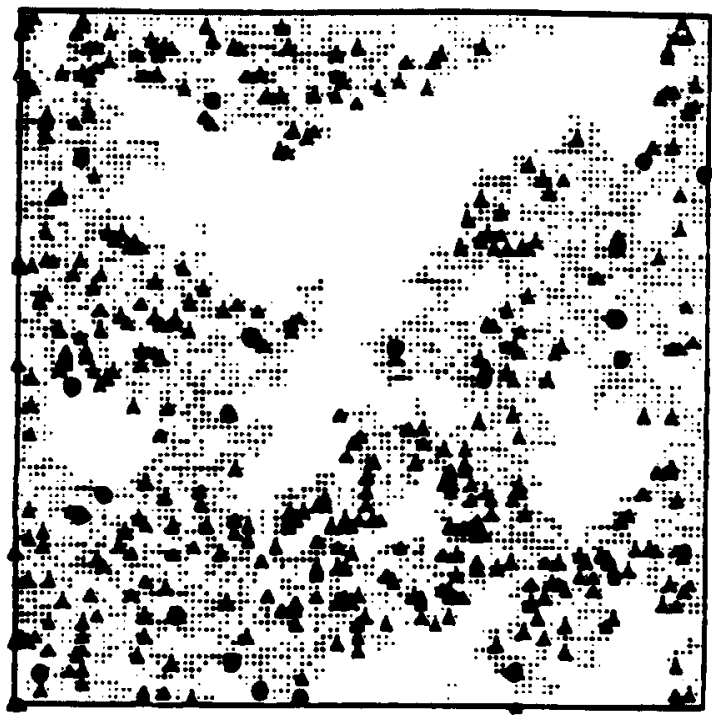

Fig. 2. Distribution of reactants for two different trap concentrations on a percolation cluster at criticality. The traps are the black circles. On Fig. $2 a$ the trap concentration is 0.05 . On Fig. $2 \mathrm{~b}$ the trap concentration is 0.005 .

$X=1.47 \pm 0.02$, with respect to the trap density $c$. This is in excellent agreement with the predictions of eq. (2): $Y=1$ and $X=2 / d_{\mathrm{s}}=1.465\left(d_{\mathrm{s}}=\right.$ 1.365). Similarly, the simulations for the critical percolation cluster are in excellent agreement with the eq. (2) predictions $Y=1$ and $X=2 / d_{s}=1.5$ $\left(d_{\mathrm{s}}=4 / 3\right)$. In addition, the depletion zones around the traps can be seen qualitatively in Fig. 2 .
SIMULATIONS OF A TRANSIENT A $+\mathbf{A} \rightarrow 0$ AND A + B $\rightarrow 0$

We have employed three types of landing relationships: correlated, random and evenly spaced landings. When a particle is added to a site occupied by another particle, the landing particle may immediately try to land on amother empty site, which is called 'forced landing'. Particles randomly move on a lattice.

Correlated landing occurs when a pair of particles lands simultaneously, separated by a certain number of lattice spacings $(\eta)$. One particle of the pair randomly finds an empty site on which to land; then the other particle chooses a site in a random direction at the correlation length distance from the first particle. If this selected site for the second particle is occupied, both particles of this pair will repeat the process described above until they find two empty sites at the correlated distance.

Random landing occurs when two particles of a pair are independent of each other, and all sites in a lattice have equal probability for a particle to land. Effectively there are no 'pairs'.

Evenly spaced landing is used only in simulations of transient reactions. Particles are distributed throughout the lattice, and have an equal distance between each other. This interval is equal to $L / N_{0}$ and is chosen to be integer, where $L$ is the lattice length and $N_{0}$ is the number of the particles at $t=0$.

Since the kinetic equation can be written for long times,

$\rho \sim t^{-\alpha}$

the kinetic data is plotted as $\ln \rho$ vs. $\ln t$. The least linear square fit is applied to find the slope of each part of each line, which is equal to $-\alpha$ in eq. (4).

\section{Correlated landing for $A+B \rightarrow 0$}

A. For $\eta=1$ : Two kinds of landing are investigated. One is a pair of particles of $A B$ with a definite orientation (e.g., AB AB AB...). The other one is a pair of particles of $A B$ with random orientations (e.g., AB AB BA...). These two cases 


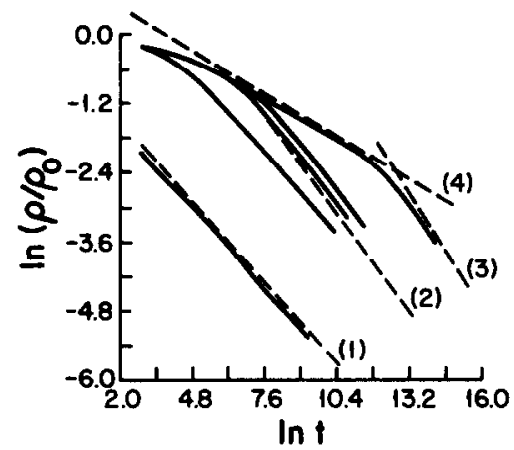

Fig. 3. $\ln \rho / \rho_{0}$ vs. In $t$ for $\mathrm{A}+\mathrm{B} \rightarrow 0$ transient reaction on one-dimensional lattice ( 30000 sites) with $\rho_{0}=0.05$. From top to bottom, the correlated landing lengths are $1000,100,64,16$, and 1 . The dashed lines are fitting lines: (1) with the slope 0.5 , (2) with the slope 0.6 , (3) with the slope 0.7 , and (4) with the slope 0.25 .

have shown the same result - a straight line with a slope 0.5. It is important to notice that this result is the same as that in the $\mathbf{A}+\mathbf{A} \rightarrow 0$ case (see below).

B. For $\eta>1$ : The slopes of the lines increase (from a value 0.25 ) after $t>\eta^{2}$ (see Fig. 3), which is considered to be the effect of correlation in landing processes. As $\eta$ increases, the slopes, at long times, increase toward the value 0.75 .

For $\eta>1$, there is no finite size effect, i.e., no second transition of the slope was found (see the bottom curve in Fig. 4).

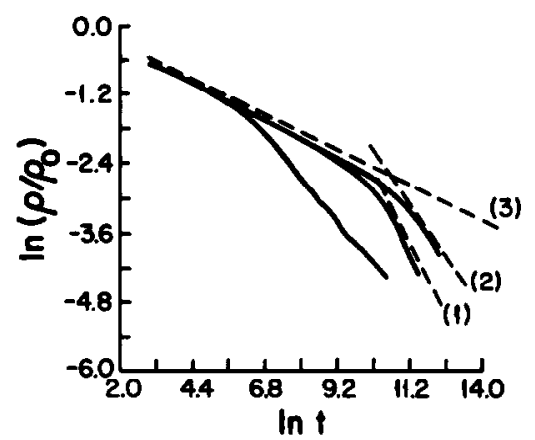

Fig. 4. $\ln \rho / \rho_{0}$ vs. $\ln t$ for $A+B \rightarrow 0$ transient reaction on one-dimensional lattice (1000 sites) with $\rho_{0}=0.20$. From top to bottom, they are random landing with reflecting boundary condition, random landing with periodic boundary condition, and correlated landing with the correlated landing lengths 50 . The dashed lines are fitting lines: (1) with the slope 1.0, (2) with the slope 0.75 , and (3) with the slope 0.25 .
Random landing for $A+B \rightarrow 0$

Two types of boundary conditions are applied: periodic and reflecting boundary conditions. In both cases, the slopes increase (from the value 0.25 ) at long times (see Fig. 4), which is considered to be a finite size effect. However, important differences between these cases were observed. The $\alpha$-value is higher with periodic boundary conditions $(\sim 1.0)$ than with reflecting boundary conditions $(\sim 0.75)$.

$A+A \rightarrow O$

Both random landing and correlated landing processes are simulated. Under the periodic boundary conditions, neither the effect of correlated landing nor the finite size effect can be found in the $\mathbf{A}+\mathbf{A} \rightarrow 0$ case (see top two lines in Fig. 3); straight lines are found with the slope 0.50 . However, under reflecting boundary conditions, at long time, a slight deviation from the slope 0.5 is observed.

Our results essentially agree with preliminary continuum models [14], replacing the ZeldovichKang-Redner time exponent $-d_{\mathrm{s}} / 4$ (for $d_{\mathrm{s}} \leqslant 4$ ) with $-\left(d_{s}+2\right) / 4$ (for $d_{s} \leqslant 2$ ), for tightly correlated systems or finite-sized lattices. However, they emphasize the relative importance of the average interparticle distance and the finite scale of the lattice or of the correlation in the source. In particular, for geminate landing, we do not observe a change in slope at late times.

\section{ACKNOWLEDGEMENTS}

This work was supported by NSF Grants No. 8842001, DMR 8815008 and DMR 8801120.

\section{REFERENCES}

1 R. Kopelman, Exciton percolation in molecular alloys and aggregates, Topics in Applied Physics, 15 (1976) 297-346.

2 D.L. Huber, D.S. Hamilton and B. Barnett, Time-dependent effects in fluorescent line narrowing, Physical Review $B, 16$ (1977) 4642-4650. 
3 M.D. Donsker and S.R.S. Varadhan, On the number of distinct sites visited by a random walk, Communications in Pure and Applied Mathematics, 32 (1979) 721-747.

4 J. Klafter, A. Blumen and G. Zumofen, Fractal behavior in trapping and reaction: a random walk study, Journal of Statistical Physics, 36 (1984) 561-577.

5 R. Kopelman, Fractal reaction kinetics, Science, 241 (1988) 1620-1626.

6 A.A. Ovchinnikov and Ya.B. Zeldovich, Role of density fluctuations in bimolecular reaction kinetics, Chemical Physics, 28 (1978) 215-218.

7 D. Toussaint and F. Wilczek, Particle-antiparticle annihilation in diffusive motion, Journal of Chemical Physics, 78 (1983) 2642-2647.

8 J. Klafter, A. Blumen and G. Zumofen, Fractal behavior in trapping and reaction: a random walk study, Journal of Statistical Physics, 36 (1984) 561-577.

9 K. Kang and S. Redner, Scaling approach for the kinetics of recombination processes, Physics Review Letters, 52 (1984) 955-958.
10 P.W. Klymko and R. Kopelman, Fractal reaction kinetics: exciton fusion on clusters, Journal of Physical Chemistry, 87 (1983) 4565-4567.

11 E. Clément, L. Sander and R. Kopelman, Source-term and excluded-volume effects on the diffusion-controlled $A+B$ $\rightarrow 0$ reaction in one dimension: rate laws and particle distributions, Physical Review A, 39 (1989) 6455-6465.

12 E. Clement, L. Sander and R. Kopelman, Steady-state diffusion-controlled $A+B \rightarrow 0$ reactions in two and three dimensions: rate laws and particle distributions, Physical Review A, 39 (1989) 6466-6471.

13 E. Clement, L. Sander and R. Kopelman, Steady-state diffusion controlled $\mathbf{A}+\mathbf{A} \rightarrow 0$ reaction in Euclidean and fractal dimensions: rate laws and particle self-ordering, Physical Review A, 39 (1989) 6472-6477.

14 K. Lindenberg, B.J. West and R. Kopelman, Diffusionlimited $\mathbf{A}+\mathbf{B} \rightarrow 0$ reaction: correlated initial condition, Physical Review A, 42 (1990) 890-894. 